GE MEASUREMENT\& CONTROL

\title{
Kontinuierliche 3D-Qualitätskontrolle mit kurzen Taktzeiten für die industrielle Fertigung
}

Bisher bleibt der industrielle Einsatz von 3DComputertomographie (CT) vor allem auf die zerstörungsfreie Prüfung ausgewählter Bauteile, die Fehlersuche sowie die Labors der F\&E beschränkt. Da eine einzelne Aufnahme - je nach der geforderten Auflösung - mit gängigen Tomographen mehrere Stunden dauern kann, war an eine 100-prozentige 3D-Qualitätskontrolle von Komponenten am Band bisher nicht zu denken. Hier bahnt sich nun möglicherweise eine Umwälzung an: Auf dem 8. X-ray Forum in Ahrensburg bei Hamburg hat GE Measurement \& Control (MC) den neuen Computertomographen „speedscan atlineCT" vorgestellt. Mit einer typischen Taktzeit von rund einer Minute pro untersuchtem Bauteil ermöglicht die verwendete Helix-MultizeilenCT-Technik laut Dr. Oliver Brunke, Product Manager CT Systems von GE MC in Wunstorf, eine kontinuierliche 3D-Qualitätskontrolle in der Fertigung.

Trotz der hohen Geschwindigkeit erkennt das speedscan-Gerät nach Herstellerangaben Porositäten ab 0,5 mm Größe, abhängig von den Abmessungen des Prüflings. Mit maximal 140 kV Leistung und hochsensiblen Detektoren durchdringt das Gerät laut GE bis zu 300 mm Aluminium. Auch die Vermessung des Bauteils (3D-Metrologie) kann in die Qualitätskontrolle integriert sein. Brunke bezifferte die Wiederholgenauigkeit des speedscan-Systems mit $\pm 10 \mu \mathrm{m}$ auf der Z-Achse sowie lediglich \pm 4 $\mu \mathrm{m}$ auf der $X$ - und Y-Achse. Ermittelt wurden diese Werte beispielhaft an einem Prüfling mit 470 mm Länge. Aktuell befindet sich das speedcsan-System bei einem großen Fahrzeughersteller in der Evaluierung. Es wird dort für die Qualitätskontrolle von Zylinderköpfen getestet.

Neben der drastischen Verkürzung der Taktzeiten für CT-Untersuchungen stand auf dem 8. X-ray Forum die hochauflösende CTTechnik im Vordergrund. So wurde im Rahmen der Technologie- und Anwenderbe- richte auf der dreitägigen Fachveranstaltung beispielsweise der Einsatz des GE nanotom $m$ in der Entwicklung für Kompositwerkstoffen auf Textilbasis vorgestellt. Hermann Finckh vom Institut für Textil- und Verfahrenstechnik (ITV) erklärte, wie das CT-System in Denkendorf genutzt wird, um den Faserverlauf von technischen Geweben, etwa aus GFK oder Aramidfasern, zu untersuchen. Das Ziel dieser Forschung ist es, in Form von CTDaten die Grundlage für Mikromodelle des Textilverhaltens unter Last (Crash-Fall) zu entwickeln. Besondere Herausforderungen liegen hier in der schwachen Absoption der Materialien bei gleichzeitig feiner Faserstruktur. Das nanotom $m$ stellt für solche Anforderungen eine Auflösung von Details $\geq 200$ $\mathrm{nm}(0,2 \mu \mathrm{m})$ zur Verfügung.

Finckh sagte, auf diesem Weg solle es künftig möglich werden, das Verhalten von Geweben unter Zug, Druck, Reibung, Biegung, Scherung sowie nach dreidimensionaler Verformung (Draping) über einem Formkörper mit einer vergleichbaren Zuverlässigkeit vorauszusagen, wie das heute bei Stahl möglich ist. Vor allem das Thema Draping beschäftigt den für seine Forschungsarbeiten bereits mehrfach ausgezeichneten Fachmann des ITV.

Anhand von aktuellen CT-Aufnahmen erläuterte Finckh den Einfluss von Faltenwürfen und Zugverschiebungen im Gewebe auf dessen Matrix und damit seine Eigenschaften. Unterstützt wird die ITV-Entwicklung von Volume Graphics als Softwarepartner. Das Heidelberger Unternehmen ist auf die Entwicklung von Software zur Erfassung und Auswertung von CT-Voxel-Datensätzen (Voxel $=$ Volumenpixel) spezialisiert und bietet mit VGStudio eine weit verbreitete Lösung an. Aus der Zusammenarbeit mit dem ITV geht zurzeit ein neues Modul für die Untersuchung von Faserverläufen in Kompositen hervor, das künftig optionaler Bestandteil von VGStudio MAX Version 2.2 sein soll.
Ein weiteres Highlight des Forums war ein Einblick in die Nutzung des weltweit größten industriellen Tomografen von GE MC am Institut für Leichtbau und Kunststofftechnik (ILK) der Technischen Universität Dresden. Prof. Hans-Jürgen Ullrich berichtete über den kombinierten Einsatz der GroßCT-Anlage vtomex L 450 mit einem Gesamtgewicht von rund 70 t sowie einem nanoCT Zusatzmodul für die Untersuchung von Leichtbaubauteilen. Ein Schwerpunkt der Untersuchungen sind Schadensmechanismen an Faserverbundwerkstoffen. Dazu werden Carbonfaser-Epoxidharzprüflinge mit unterschiedlicher Faserstruktur innerhalb der CT-Anlage bis zum Versagen mit Zug und Druck belastet und dabei die Schadensentwicklung im Detail beobachtet. Aufbauend auf dieser Phänomenologie sollen Modelle zur Schadbildausprägung der Werkstoffe entwickelt werden. jc $\mathbf{0}$

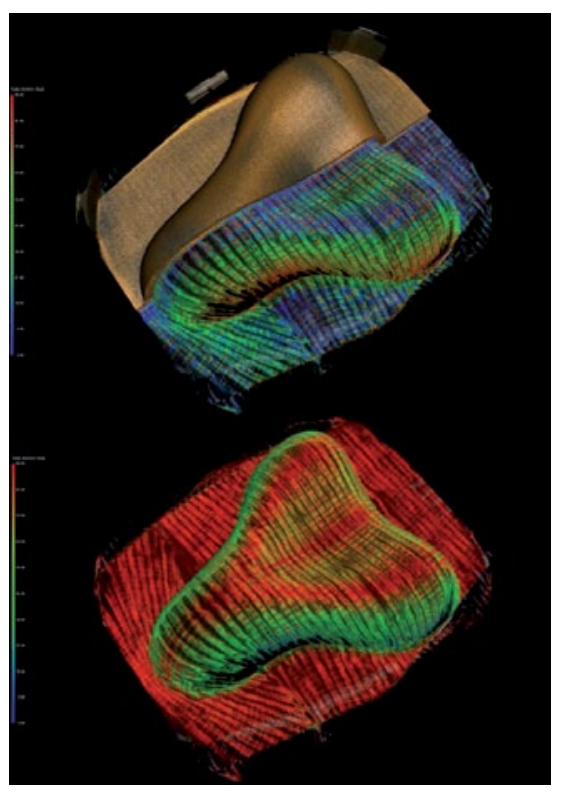

Faserverlaufsanalyse am ITV in Denkendorf mittels hoch aufgelöstem CT Scan; das Beispiel zeigt ein in einer 3D-Form drapiertes, unidirektionales Carbonfasergewebe 